\title{
Programas de fertilización química sobre líneas promisorias de arroz (Oryza sativa L.)
}

\section{Chemical fertilization programs on promissory rice lines (Oryza sativa L.)}

\author{
Yari Ruiz $^{1 *}$, Guillermo Villacrés ${ }^{1}$, Gino Galarza ${ }^{2}$, Carlos Valarezo Beltrón ${ }^{3}$ \\ ${ }^{1}$ Universidad Técnica de Babahoyo \\ 2 Instituto Superior Técnico Eugenio Espejo \\ ${ }^{3}$ Escuela Superior Politécnica Agropecuaria de Manabí \\ *yruiz@utb.edu.ec
}

DOI: https://doi.org/10.26871/killkana_tecnica.v3i3.574

\begin{abstract}
Resumen
El presente trabajo de investigación se llevó a cabo en la Facultad de Ciencias Agropecuarias de la Universidad Técnica de Babahoyo, la cual se encuentra ubicada en el Km $7 \frac{1}{2} 2$ de la vía Babahoyo - Montalvo. Los objetivos principales del estudio que se muestra en este artículo, son: evaluar el efecto de la fertilización química en base al análisis de suelo en el cultivo de arroz; identificar el genotipo de mayor rendimiento y analizar desde el punto de vista económico los tratamientos para la fertilización química. Se utilizaron tres tratamientos (material genético), como INIAP 15, Líneas GO37647, GO39845 y cuatro subtratamientos (niveles de fertilización), a base de nitrógeno (urea), potasio (muriato de potasio) y Zinc (Zinquell), con cuatro repeticiones. Se utilizó el diseño de parcelas divididas, con tres tratamientos, cuatro subtratamientos y cuatro repeticiones. Para la comparación de los promedios de las variables, se empleó la prueba de Rangos Múltiples de Duncan al $5 \%$ de probabilidad. En base a los resultados alcanzados en el presente estudio, se concluye que para la altura de plantas, la longitud de panículas, la floración y la maduración del cultivo, se obtuvo alta significación estadística solo para el material genético; para el número de macollos por $\mathrm{m}^{2}$ y granos por panícula fueron significativos al $95 \%$ de probabilidades, tanto para material genético, como para fertilización; mientras que el testigo sin fertilización alcanzó solo 2.47 ton/ha. El suelo presentó déficit principalmente de nitrógeno y en menor intensidad de $\mathrm{K}$ y Zn; mientras en la calidad de grano sobresalió la línea GO39845 con 68 \% de arroz pilado; y el análisis químico del suelo constituyó una buena herramienta para la fertilización del cultivo.
\end{abstract}

Palabras clave: arroz, fertilización, análisis de suelo, producción.

\begin{abstract}
This research was carried out at the Technical University of Babahoyo, Agricultural Sciences Faculty, located at $71 / 2$ $\mathrm{Km}$ from the Babahoyo-Montalvo road. The main objectives of the study are: To evaluate the chemical fertilization effect, based on the rice crops' soil analysis, to identify the highest production genotype, and to analyze the chemical fertilization treatments from the economic point of view. Three treatments (genetic material), such as INIAP 15, GO37647, and GO39845 lines were used, as well as four sub-treatments (fertilization levels) with four repetitions, based on nitrogen (urea), potassium (muriate of potassium) and Zinc (Zinquell). The divided plot design, with three treatments, four subtreatments, and four repetitions was used. The Duncan's Multiple Range Test, at 5\% probability, was utilized for the comparison of variables' averages. Based on the results obtained in this study, it can be concluded that for plant height, panicle length, flowering, and crop maturation, high statistical significance was obtained for genetic material; the number of stalks per $m^{2}$, and grains per panicle had a $95 \%$ significance, both for genetic material and for fertilization; while the control with no fertilization reached only 2.47 ton /ha. The soil was mainly lacking in nitrogen, and to a lesser extent in $K$ and $\mathrm{Zn}$. In terms of grain quality, the GO39845 with peeled rice surpassed the line by 68\%, and the soil chemical analysis was a good tool for crop fertilization.
\end{abstract}

Keywords: rice, fertilization, soil analysis, production.

\section{INTRODUCCIÓN}

El aumento de la producción de arroz en América Latina es una necesidad prioritaria para asegurar el suministro del grano a la población cada vez mayor. Para garantizar la disponibilidad de este alimento básico a precios favorables para todos los extractos sociales, se requiere que el 
agricultor cuente con nuevas variedades, los conocimientos para mejorar la producción, aumentar la productividad y la rentabilidad del cultivo a nivel de campo [1].

El cultivo de arroz en nuestro país es de gran importancia socioeconómica, según datos registrados se cultivan alrededor de 343.936 hectáreas, la mayoría de esta superficie está en manos de pequeños productores que desarrollan el cultivo mediante la aplicación de diversas tecnologías, que están en relación con la disposición de recursos económicos, acceso a la capacitación y al incentivo de los precios del mercado [2].

Las características físico-químicas del suelo, deben ser conocidas por el productor agrícola, ya que el crecimiento y desarrollo de los cultivos y la cantidad y calidad de las cosechas están en relación directa con los nutrimentos y las características de los suelos. El rendimiento de un cultivo es afectado por diversos factores, entre los que ocupa un lugar importante la disponibilidad de los nutrimentos esenciales para las plantas en el suelo. Cuando estos nutrimentos no están en cantidades adecuadas, hay necesidad de adicionar fertilizantes químicos o enmiendas para suplir las necesidades y corregir condiciones adversas. Desde este punto de vista, el análisis químico del suelo puede suministrar información muy valiosa. [3].

El principal problema de bajo rendimiento del cultivo de arroz es indispensablemente por el desconocimiento de las cantidades de fertilizantes disponibles en el suelo, siendo asimilables por la planta y la escasa utilización de variedades mejoradas. [4].

Por las razones antes expuestas, se justifica la presente investigación para determinar los niveles de fertilización adecuados en la variedad de arroz INIAP 15 y dos líneas promisorias de arroz en la zona de Babahoyo. [5].

\section{MATERiales y MÉtodos}

El presente trabajo de investigación se llevó a cabo en la Facultad de Ciencias Agropecuarias de la Universidad Técnica de Babahoyo, ubicada en el $\mathrm{Km} 7$ 1/2 de la vía Babahoyo - Montalvo.

El terreno se encuentra en las coordenadas geográficas de $79^{\circ} 32^{\prime}$ Latitud Sur, y $1^{\circ} 49^{\prime}$ de Latitud Oeste, con una altura de $8 \mathrm{msnm}$, presenta un clima tropical húmedo, con temperatura media anual de $25.5^{\circ} \mathrm{C}$, una precipitación media anual de $2329.00 \mathrm{~mm}$, humedad relativa de $82 \%$ y 987.1 horas de heliofanía promedio anual. El suelo es de topografía plana, textura franco arcillosa y drenaje regular [6].

Se utilizaron las líneas GO37647, 39845 y para compararlas se empleó una variedad común como lo es la INIAP 15.

Se utilizaron tres tratamientos (material genético), cuatro subtratamientos (niveles de fertilización) con cuatro repeticiones, las características de los tratamientos y subtratamientos estudiados.
TABLA I: Tratamientos y subtratamientos estudiados, en el efecto de la fertilización química en base al análisis de suelo en la variedad INIAP 15 y dos líneas promisorias de arroz en la zona de Babahoyo

\begin{tabular}{lccc}
\hline Tratamientos & \multicolumn{3}{c}{ Subtratamiento } \\
\cline { 2 - 4 } & $\mathrm{N}(\mathrm{kg} / \mathrm{ha})$ & $\begin{array}{c}\mathrm{K}_{2} \mathrm{O} \\
(\mathrm{kg} / \mathrm{ha})\end{array}$ & $\begin{array}{c}\mathrm{Zn} \\
(\mathrm{kg} / \mathrm{ha})\end{array}$ \\
\hline \multirow{3}{*}{ Iniap 15 } & 160 & 0 & 0 \\
& 160 & 90 & 0 \\
& 160 & 90 & 2 \\
Línea GO37647 & 100 (testigo) & 0 & 0 \\
\hline \multirow{3}{*}{ Línea GO39845 } & 160 & 0 & 0 \\
& 160 & 90 & 0 \\
& 160 & 90 & 2 \\
& 100 (testigo) & 0 & 0 \\
\hline & 160 & 0 & 0 \\
& 160 & 90 & 0 \\
& 160 & 90 & 2 \\
& 100 (testigo) & 0 & 0 \\
\hline
\end{tabular}

Se utilizó el diseño Parcelas divididas, con tres tratamientos, cuatro subtratamientos y cuatro repeticiones. Para la comparación de los promedios de las variables, se utilizó la prueba de Rangos Múltiples de Duncan al $5 \%$ de probabilidad.

En cuanto a prácticas agronomías, el ensayo se manejo igual en las subparcelas donde se sembraron los materiales genéticos, con excepción de las dosis de fertilizantes que se aplicaron para la nutrición de los materiales cultivados fraccionados en partes proporcionales a los 15, 30 y 45 días después de la siembra.

\section{RESUltados}

\section{A. Altura de planta}

La mayor altura de planta a la cosecha (Tabla II), se observó en la línea GO39845 (65.16 cm), superior estadísticamente a la línea GO37647 $(60.20 \mathrm{~cm})$ e INIAP 15 $(55.30 \mathrm{~cm})$; para la fertilización, la aplicación de $100 \mathrm{~kg} \mathrm{~N}$ $(62.19 \mathrm{~cm})$ presentó el mayor valor de altura de planta, con igualdad estadística a $160 \mathrm{~kg} \mathrm{~N}+90 \mathrm{~kg} \mathrm{~K} 2 \mathrm{O}+21 \mathrm{Zn}(61.52$ $\mathrm{cm}) ; 160 \mathrm{~kg} \mathrm{~N}+90 \mathrm{~kg} \mathrm{~K} \mathrm{O}_{2} \mathrm{O}(60.12 \mathrm{~cm})$ y superiores a 160 $\mathrm{kg} \mathrm{N}(57.05 \mathrm{~cm})$

TABLA II: Altura de planta a la cosecha ( $\mathrm{cm})$, en efecto de la fertilización química en base a los resultados del análisis de suelo en la variedad INIAP 15 y dos líneas promisorias de arroz

\begin{tabular}{|c|c|c|c|c|c|c|}
\hline \multicolumn{3}{|c|}{$\begin{array}{c}\text { Subtratamientos } \\
\text { (Niveles de Fertilización) }\end{array}$} & \multicolumn{3}{|c|}{$\begin{array}{c}\text { Tratamientos } \\
\text { (Material Genético.) }\end{array}$} & \multirow[t]{2}{*}{ Media ns } \\
\hline$\underset{(\mathrm{kg} / \mathrm{ha})}{\mathrm{N}}$ & $\begin{array}{c}\mathrm{K}_{2} \mathrm{O} \\
(\mathrm{kg} / \mathrm{ha})\end{array}$ & $\begin{array}{c}\mathrm{Zn} \\
(\mathrm{l} / \mathrm{ha})\end{array}$ & Iniap 15 & $\begin{array}{c}\text { Línea } \\
\text { GO } 37647\end{array}$ & $\begin{array}{c}\text { Línea } \\
\text { GO } 39845\end{array}$ & \\
\hline 160 & 0 & 0 & 46.63 & 62.98 & 61.55 & $57.05 \mathrm{~b}$ \\
\hline 160 & 90 & 0 & 53.32 & 55.90 & 71.13 & $60.12 a b$ \\
\hline 160 & 90 & 2 & 56.59 & 61.86 & 66.10 & $61.52 \mathrm{ab}$ \\
\hline 100 (testigo) & 0 & 0 & 64.65 & 60.08 & 61.85 & 62.19 a \\
\hline \multicolumn{3}{|l|}{ Media ** } & $55.30 \mathrm{c}$ & $60.20 \mathrm{~b}$ & $65.16 \mathrm{a}$ & 60.22 \\
\hline \multicolumn{7}{|c|}{ Coeficiente de variación 8.63\% } \\
\hline
\end{tabular}


El mayor promedio de macollos/m2 se alcanzó en la línea promisoria GO37647 con 345.75 macollos y el menor valor INIAP 15 con 311.15 macollos; en cuanto a los niveles de fertilizantes, la utilización de $160 \mathrm{~kg} \mathrm{~N}+90$ $\mathrm{kg} \mathrm{K}_{2} \mathrm{O}$ con 343.75 macollos presentó el mayor valor y el menor valor $100 \mathrm{~kg} \mathrm{~N}$, con 304.00 macollos, como se observa en la Tabla III.

TABLA III: Macollos/m2, en efecto de la fertilización química en base a los resultados del análisis de suelo en la variedad INIAP 15 y dos líneas promisorias de arroz

\begin{tabular}{|c|c|c|c|c|c|c|}
\hline \multicolumn{3}{|c|}{$\begin{array}{c}\text { Subtratamientos } \\
\text { (Niveles de Fertilización) }\end{array}$} & \multicolumn{3}{|c|}{$\begin{array}{c}\text { Tratamientos } \\
\text { (Material Genético.) }\end{array}$} & \multirow[t]{2}{*}{ Media ${ }^{\text {ns }}$} \\
\hline $\begin{array}{c}\mathbf{N} \\
(\mathrm{kg} / \mathrm{ha})\end{array}$ & $\begin{array}{c}\mathrm{K}_{2} \mathrm{O} \\
(\mathrm{kg} / \mathrm{ha})\end{array}$ & $\begin{array}{c}\text { Zn } \\
(\mathrm{I} / \mathrm{ha})\end{array}$ & Iniap 15 & $\begin{array}{c}\text { Línea } \\
\text { GO } 37647\end{array}$ & $\begin{array}{c}\text { Línea } \\
\text { GO } 39845\end{array}$ & \\
\hline 160 & 0 & 0 & 329.50 & 343.50 & 323.50 & 332.17 \\
\hline 160 & 90 & 0 & 352.50 & 358.50 & 320.25 & 343.75 \\
\hline 160 & 90 & 2 & 332.50 & 323.00 & 328.75 & 328.08 \\
\hline 100 (testigo) & 0 & 0 & 230.50 & 358.00 & 323.50 & 304.00 \\
\hline \multicolumn{3}{|l|}{ Media $^{\text {ns }}$} & 311.25 & 345.75 & 324.00 & 327.00 \\
\hline \multicolumn{7}{|c|}{ Coeficiente de variación $15.00 \%$} \\
\hline
\end{tabular}

\section{B. Panículas $/ m^{2}$}

En la Tabla IV se muestra que el mayor promedio de panículas/m2 presentó la línea de arroz GO39845 con 302.13 panículas, igual estadísticamente al promedio de la línea GO37647, con 295.39 panículas y superiores a la variedad INIAP 15, con 256.31 panículas. En niveles de fertilización, la aplicación de $160 \mathrm{~kg} \mathrm{~N}+90 \mathrm{~kg} \mathrm{~K} 2 \mathrm{O}$ presentó el mayor valor (305.83 panículas $/ \mathrm{mm}^{2}$ ), igual estadísticamente a 160 $\mathrm{kg} \mathrm{N}+90 \mathrm{~kg} \mathrm{~K} \mathrm{O}_{2} \mathrm{O}+21 \mathrm{Zn}\left(291.39\right.$ panículas $\left./ \mathrm{mm}^{2}\right) ; 100 \mathrm{~kg}$ $\mathrm{N}\left(280.18\right.$ panículas $\left./ \mathrm{mm}^{2}\right)$ y superiores a $160 \mathrm{~kg} \mathrm{~N}(261.03$ panículas $\left./ \mathrm{mm}^{2}\right)$.

TABLA IV: Panículas $/ \mathrm{m}^{2}$, en efecto de la fertilización química en base a los resultados del análisis de suelo en la variedad INIAP 15 y dos líneas promisorias de arroz

\begin{tabular}{|c|c|c|c|c|c|c|}
\hline \multicolumn{3}{|c|}{$\begin{array}{c}\text { Subtratamientos } \\
\text { (Niveles de Fertilización) }\end{array}$} & \multicolumn{3}{|c|}{$\begin{array}{c}\text { Tratamientos } \\
\text { (Material Genético.) }\end{array}$} & \multirow[t]{2}{*}{ Media * } \\
\hline $\begin{array}{c}N \\
(k g / h a)\end{array}$ & $\begin{array}{c}\mathrm{K}_{2} \mathbf{O} \\
(\mathbf{k g} / \mathbf{h a})\end{array}$ & \begin{tabular}{|c|} 
Zn \\
$(\mathbf{l} / \mathbf{h a})$
\end{tabular} & Iniap 15 & $\begin{array}{c}\text { Línea } \\
\text { Go } 37647\end{array}$ & $\begin{array}{c}\text { Línea } \\
\text { GO } 39845\end{array}$ & \\
\hline 160 & 0 & 0 & 228.75 & 281.08 & 273.28 & $261.03 b$ \\
\hline 160 & 90 & 0 & 294.00 & 326.50 & 297.00 & 305.83 a \\
\hline 160 & 90 & 2 & 288.00 & 279.93 & 306.25 & $291.39 \mathrm{ab}$ \\
\hline 100 (testigo) & 0 & 0 & 214.50 & 294.05 & 332.00 & $280.18 \mathrm{ab}$ \\
\hline \multicolumn{3}{|l|}{ Media * } & $256.31 \mathrm{~b}$ & 295.39 a & 302.13 a & 284.61 \\
\hline \multicolumn{7}{|c|}{ Coeficiente de variación $12.26 \%$} \\
\hline
\end{tabular}

Los promedios de granos por panícula se presentan en la Tabla V, el análisis de varianza reportó diferencias significativas para material genético y niveles de fertilizantes. El coeficiente de variación fue $16.33 \%$.

El mayor número de granos por panícula se obtuvo en la línea GO37647 con 119.72 granos, igual estadísticamente a la línea GO39845, con 110.78 granos y superiores a INIAP
15, con 96.08 granos; en niveles de fertilización, el mayor promedio lo obtuvo la utilización de $100 \mathrm{~kg} \mathrm{~N}$, con 117.59 granos, igual estadísticamente a la aplicación de $160 \mathrm{~kg} \mathrm{~N}$ + $90 \mathrm{~kg} \mathrm{~K}_{2} \mathrm{O}$ y $160 \mathrm{~kg} \mathrm{~N}+90 \mathrm{~kg} \mathrm{~K}{ }_{2} \mathrm{O}+21 \mathrm{Zn}$, con 115.42 y 104.96 granos, respectivamente.

TABLA V: Granos/panículas, en efecto de la fertilización química en base a los resultados del análisis de suelo en la variedad INIAP 15 y dos líneas promisorias de arroz

\begin{tabular}{|c|c|c|c|c|c|c|}
\hline \multicolumn{3}{|c|}{$\begin{array}{c}\text { Subtratamientos } \\
\text { (Niveles de Fertilización) }\end{array}$} & \multicolumn{3}{|c|}{$\begin{array}{c}\text { Tratamientos } \\
\text { (Material Genético.) }\end{array}$} & \multirow[t]{2}{*}{ Media * } \\
\hline $\begin{array}{c}\mathrm{N} \\
(\mathrm{kg} / \mathrm{ha})\end{array}$ & $\begin{array}{c}\mathrm{K}_{2} \mathrm{O} \\
(\mathrm{kg} / \mathrm{ha})\end{array}$ & \begin{tabular}{|c|} 
Zn \\
$($ I/ha $)$
\end{tabular} & Iniap 15 & $\begin{array}{c}\text { Línea } \\
\text { Go } 37647\end{array}$ & $\begin{array}{c}\text { Línea } \\
\text { GO } 39845\end{array}$ & \\
\hline 160 & 0 & 0 & 79.35 & 126.90 & 86.18 & $97.48 \mathrm{~b}$ \\
\hline 160 & 90 & 0 & 105.63 & 121.50 & 119.13 & 115.42 a \\
\hline 160 & 90 & 2 & 77.10 & 116.53 & 121.25 & $104.96 \mathrm{ab}$ \\
\hline 100 (testigo) & 0 & 0 & 122.25 & 113.95 & 116.58 & 117.59 a \\
\hline \multicolumn{3}{|l|}{ Media * } & $96.08 \mathrm{~b}$ & 119.72 a & $110.78 \mathrm{a}$ & 108.86 \\
\hline \multicolumn{7}{|c|}{ Coeficiente de variación $16.33 \%$} \\
\hline
\end{tabular}

En la Tabla VI, se muestran los promedios de peso de 1000 granos, el mayor peso de granos lo presentó la línea promisoria GO39845, con $26.70 \mathrm{~g}$, superior estadísticamente a los demás tratamientos, siendo INIAP 15 la de menor valor, con $23.91 \mathrm{~g}$. En niveles de fertilización, el mayor promedio con $27.21 \mathrm{~g}$ lo obtuvo el uso de $160 \mathrm{~kg}$ $\mathrm{N}+90 \mathrm{~kg} \mathrm{~K} \mathrm{~K}_{2} \mathrm{O}+21 \mathrm{Zn}$, superior estadísticamente al resto de subtratamientos, presentándose en la aplicación de 100 $\mathrm{kg} \mathrm{N}$ el menor valor, con $24.00 \mathrm{~g}$.

TABLA VI: Peso de 1000 granos (g), en efecto de la fertilización química en base a los resultados del análisis de suelo en la variedad INIAP 15 y dos líneas promisorias de arroz

\begin{tabular}{|c|c|c|c|c|c|c|}
\hline \multicolumn{3}{|c|}{$\begin{array}{c}\text { Subtratamientos } \\
\text { (Niveles de Fertilización) }\end{array}$} & \multicolumn{3}{|c|}{$\begin{array}{c}\text { Tratamientos } \\
\text { (Material Genético.) }\end{array}$} & \multirow[t]{2}{*}{ Media ** } \\
\hline $\begin{array}{c}\mathrm{N} \\
(\mathrm{kg} / \mathrm{ha})\end{array}$ & $\begin{array}{c}\mathrm{K}_{2} \mathrm{O} \\
(\mathrm{kg} / \mathrm{ha})\end{array}$ & \begin{tabular}{|c|} 
Zn \\
$(\mathrm{l} / \mathrm{ha})$
\end{tabular} & Iniap 15 & \begin{tabular}{|c|} 
Línea \\
GO 37647
\end{tabular} & $\begin{array}{c}\text { Línea } \\
\text { GO } 39845\end{array}$ & \\
\hline 160 & 0 & 0 & 23.57 & 23.91 & 23.36 & $24.61 \mathrm{c}$ \\
\hline 160 & 90 & 0 & 23.88 & 25.31 & 26.72 & $25.30 \mathrm{~b}$ \\
\hline 160 & 90 & 2 & 25.19 & 27.18 & 29.26 & $27.21 \mathrm{a}$ \\
\hline 100 (testigo) & 0 & 0 & 22.98 & 24.55 & 24.48 & $24.00 \mathrm{~d}$ \\
\hline \multicolumn{3}{|l|}{ Media ** } & $23.91 \mathrm{c}$ & $25.23 \mathrm{~b}$ & $26.70 a$ & 25.28 \\
\hline \multicolumn{7}{|c|}{ Coeficiente de variación $2.15 \%$} \\
\hline
\end{tabular}

En la Tabla VII, se presentan los resultados dee rendimiento En esta variable se reportó que la línea GO37647, obtuvo el mayor valor, con $4090.53 \mathrm{~kg} / \mathrm{ha}$, superior estadísticamente a los demás tratamientos, cuyo menor valor lo presentó INIAP 15 , con $2107.27 \mathrm{~kg} / \mathrm{ha}$, mientras que en niveles de fertilización, la aplicación de $160 \mathrm{~kg} \mathrm{~N}+90 \mathrm{~kg}$ $\mathrm{K}_{2} \mathrm{O}+21 \mathrm{Zn}$ alcanzó el mayor valor, con $3799.37 \mathrm{~kg} / \mathrm{ha}$, superior estadísticamente a los demás subtratamientos, siendo el menor valor, con $2470.69 \mathrm{~kg} / \mathrm{ha}$, el uso de $100 \mathrm{~kg} \mathrm{~N}$. 
TABLA VII: Rendimiento ( $\mathrm{kg} / \mathrm{ha}$ ), en efecto de la fertilización química en base a los resultados del análisis de suelo en la variedad INIAP 15 y dos líneas promisorias de arroz

\begin{tabular}{|c|c|c|c|c|c|c|}
\hline \multicolumn{3}{|c|}{$\begin{array}{c}\text { Subtratamientos } \\
\text { (Niveles de Fertilización) }\end{array}$} & \multicolumn{3}{|c|}{$\begin{array}{c}\text { Tratamientos } \\
\text { (Material Genético.) }\end{array}$} & \multirow[t]{2}{*}{ Media ** } \\
\hline $\begin{array}{c}\mathbf{N} \\
(\mathbf{k g} / \mathbf{h a})\end{array}$ & $\begin{array}{c}\mathrm{K}_{2} \mathrm{O} \\
(\mathrm{kg} / \mathrm{ha})\end{array}$ & $\begin{array}{c}\mathrm{Zn} \\
(\mathrm{I} / \mathrm{ha})\end{array}$ & Iniap 15 & $\begin{array}{c}\text { Línea } \\
\text { GO } 37647\end{array}$ & $\begin{array}{c}\text { Línea } \\
\text { GO } 39845\end{array}$ & \\
\hline 160 & 0 & 0 & 2229.16 & 4109.84 & 2878.78 & $3072.59 \mathrm{bc}$ \\
\hline 160 & 90 & 0 & 1676.13 & 4431.81 & 4182.19 & $3430.05 a b$ \\
\hline 160 & 90 & 2 & 3248.10 & 4667.06 & 3482.95 & $3799.37 a$ \\
\hline 100 (testigo) & 0 & 0 & 1275.70 & 3153.41 & 2982.95 & $2470.69 \mathrm{c}$ \\
\hline \multicolumn{3}{|l|}{ Media ** } & $2107.27 \mathrm{c}$ & 4090.53 a & $3381.72 b$ & 3193.17 \\
\hline
\end{tabular}
Promedios
Duncan.

En lo referente a yelen, polvillo y tamo, los mayores promedios lo obtuvieron INIAP 15 y la línea GO37647 y los menores promedios la línea GO39845, como se muestra en la Tabla VIII.

TABLA VIII: Calidad molinera en $100 \mathrm{~g}$ de muestra, en efecto de la fertilización química en base a los resultados del análisis de suelo en la variedad INIAP 15 y dos líneas promisorias de arroz

\begin{tabular}{|l|c|c|c|}
\hline \multirow{2}{*}{$\begin{array}{c}\text { Calidad } \\
\text { molinera (g) }\end{array}$} & \multicolumn{3}{|c|}{ Tratamientos } \\
\cline { 2 - 4 } & Iniap 15 & $\begin{array}{c}\text { Línea } \\
\text { GO } 37647\end{array}$ & $\begin{array}{c}\text { Línea } \\
\text { GO 39845 }\end{array}$ \\
\hline Arroz Integral & 80 & 80 & 81 \\
\hline Arroz Pilado & 65 & 66 & 68 \\
\hline Arrocillo & 5 & 4 & 3 \\
\hline Yelen & 2 & 2 & 1.5 \\
\hline Polvillo & 8 & 8 & 7.5 \\
\hline Tamo & 20 & 20 & 19 \\
\hline
\end{tabular}

\section{CONCLUSIONES}

Las líneas promisorias mostraron mejor respuesta agronómica que el tradicional arroz INIAP 15.
El arroz GO39845 obtuvo mostro resultados excelentes cuanto a calidad de grano.

\section{REFERENCIAS}

[1] S. Alcívar and S. Mestanza, "Nutrición mineral del cultivo de arroz," Manual del cultivo de arroz Instituto Nacional Autónomo de Investigaciones Agropecuarias. EE Boliche. Manual, no. 66, p. 50, 2007.

[2] A. Tropical, "Centro internacional de agrucultura tropical," 2016.

[3] Rojas, Fertilización orgánica del arroz. Ecuador: FACIAG, 2016.

[4] INIAP, "Manual de producción de arroz de calidad en el Ecuador," 2014.

[5] Cano, Evaluación del cultivo de arroz. Ecuador: FACIAG, 2016.

[6] O. Caicedo Camposano, C. Balmaseda Espinosa, and J. Proaño Saraguro, "Programación del riego del banano (Musa paradisiaca) en finca San José 2, Los Ríos, Ecuador," Revista Ciencias Técnicas Agropecuarias, vol. 24, no. 2, pp. 18-22, 2015.

Recibido: 26 de agosto de 2019

Aceptado: 22 de octubre de 2019 